\title{
ANÁLISIS DE LA EFICIENCIA ENERGÉTICA DE LA BIBLIOTECA MUNICIPAL DE CAMPO MOURÃO-PR: PARÁMETROS DEL PROGRAMA NACIONAL DE ETIQUETADO (RTQ-C)
}

\section{ARTÍCULO ORIGINAL}

CARVALHO, Fanny Islana de Lima ${ }^{1}$, HALMEMAN, Maria Cristina Rodrigues ${ }^{2}$, LERCO, Felipe Matos dos Santos ${ }^{3}$

CARVALHO, Fanny Islana de Lima. HALMEMAN, Maria Cristina Rodrigues. LERCO, Felipe Matos dos Santos. Análisis de la eficiencia energética de la biblioteca municipal de Campo Mourão-PR: parámetros del programa nacional de etiquetado (RTQ-C). Revista Científica Multidisciplinar Núcleo do Conhecimento. Año. 06, Ed. 10, Vol. 05, págs. 43-62. Octubre de 2021. ISSN: 2448-0959, Enlace de acceso: $\quad$ https://www.nucleodoconhecimento.com.br/ingenieria-ambiental-es/labiblioteca-municipal, DOI: 10.32749/nucleodoconhecimento.com.br/ingenieriaambiental-es/la-biblioteca-municipal

\section{RESUMEN}

El sector de la construcción, responsable de los impactos ambientales como la generación de residuos, el consumo de agua y energía, tiene como desafío actual llevar la eficiencia energética al sitio de construcción y al entorno post-construido. Así, en relación con el consumo energético, el INMETRO desarrolló un programa de etiquetado dirigido a clasificar la eficiencia energética de los equipos eléctricos y también de los edificios. Resulta que los edificios públicos están obligados a obtener la Etiqueta Nacional de Conservación de Energía (ENCE). Teniendo en cuenta lo anterior, se pregunta cómo medir la eficiencia energética de los edificios públicos,

\footnotetext{
${ }^{1}$ Licenciado en Arquitectura y Urbanismo. ORCID: 0000-0002-1871-2618.

2 Licenciado en Ingeniería Civil. ORCID: 0000-0002-2809-6601.

${ }^{3}$ Doctor en Ciencias Agronómicas. ORCID: 0000-0001-6964-7572.
}

RC: 100245

Disponible: https://www.nucleodoconhecimento.com.br/ingenieria-ambiental-es/labiblioteca-municipal 
haciéndolos energéticamente apropiados, y así satisfacer las necesidades del usuario, proporcionando confort ambiental y economía. El objetivo de este trabajo fue simular la obtención de la etiqueta ENCE Geral y presentar el nivel de eficiencia energética de la Biblioteca Municipal de Campo Mourão-PR. Se utilizó el Método Prescriptivo, establecido por el Reglamento Técnico de Calidad para el Nivel de Eficiencia Energética de Edificios Comerciales, de Servicios y Públicos (RTQ-C). Se analizaron tres sistemas de construcción: Revestimiento, Sistema de iluminación y Sistema de aire acondicionado. Como resultado, el edificio en estudio presentó una clasificación de eficiencia de nivel $C$, en una escala de $A$ - E, siendo A muy eficiente, y $\mathrm{E}$ ineficiente. Se concluye que el edificio no proporciona confort a los usuarios, además de un alto consumo de energía. Sin embargo, es posible adoptar medidas que pueden ayudar a optimizar el nivel de eficiencia energética en el sitio.

Palabras clave: Revestimiento, Sistema de iluminación, Aire acondicionado, Método prescriptivo, Clasificación total.

\section{INTRODUCCIÓN}

La preocupación de los países desarrollados por la conservación del medio ambiente dio lugar a conferencias internacionales, buscando alternativas económicas. (DEBATES INTERDISCIPLINARES VII, 2016).Ejemplos de estas conferencias son el Protocolo de Kyoto y el Convenio de Estocolmo. En el escenario nacional, a partir de 2014 los edificios públicos comenzaron a estar obligados a presentar etiqueta de eficiencia energética (ENCE), según lo publicado en el Boletín Oficial por la Secretaría de Logística y Tecnología de la normativa IN02/2014 (CONSELHO DE ARQUITETURA E URBANISMO/BR, 2014). En vista de lo anterior, surgió la pregunta sobre cómo es posible medir la eficiencia energética de un edificio público, garantizando el confort y la economía a los usuarios.

El Programa Brasileño de Etiquetado (PBE), junto con el PBE Edifica, surgió de discusiones iniciadas por el Instituto Nacional de Metrología, Calidad y Tecnología

RC: 100245

Disponible: https://www.nucleodoconhecimento.com.br/ingenieria-ambiental-es/labiblioteca-municipal 
(INMETRO). El objetivo de este programa es sensibilizar al consumidor a través de la información sobre el funcionamiento de los equipos y su consumo energético (JORNADA DE ESTUDIOS EN ENERGÍA ELÉCTRICA, 2019). Este programa desarrolló el Reglamento Técnico de Calidad para el Nivel de Eficiencia Energética de Edificios Residenciales (RTQ-R) y los Requisitos Técnicos de Calidad para el Nivel de Eficiencia Energética de Edificios Comerciales, de Servicio y Públicos (RTQ-C). El sistema de etiquetado mide el nivel de eficiencia energética en una escala "A - E", siendo "A" más eficiente, y "E" menos eficiente. RTQ-C evalúa edificios a través de dos métodos: Método de Simulación (realizado por software) y Método Prescriptivo (hecho a través de ecuaciones).

Existen cuatro tipos de etiquetas, que son: ENCE General (tiene en cuenta tres sistemas de construcción: revestimiento, iluminación y aire acondicionado); ENCE Parcial (evalúa de forma independiente cada sistema); ENCE Simulación (obtenida durante la fase de proyecto); y ENCE Edificio construido (se puede solicitar previa obtención del permiso de la obra o conexiones comprobadas de luz y gas por parte de los concesionarios).

Este artículo tuvo como objetivo identificar el nivel de Eficiencia Energética de la Biblioteca Municipal simulando la obtención de ENCE General. El análisis se desarrolló de acuerdo con el Manual de aplicación RTQ-C Versión 4 2017. Para lograr el objetivo general, este trabajo se desarrolló sobre la base de los siguientes objetivos específicos: Observar los requisitos previos requeridos por el Manual para la Aplicación del RTQ-C; recopilar datos sobre el sitio y procesar información de diseño arquitectónico; calcular y determinar el nivel de eficiencia energética de los tres sistemas del edificio.

RC: 100245

Disponible: https://www.nucleodoconhecimento.com.br/ingenieria-ambiental-es/labiblioteca-municipal 


\section{MATERIALES Y MÉTODOS}

\subsection{MATERIALES}

La actual Biblioteca Municipal Profesor Egydio Martello está ubicada en la zona central de Campo Mourão-PR. La ciudad tiene aproximadamente 96.102 habitantes (IBGE, 2020). Campo Mourão es un centro de COMCAM (Comunidad de Municipios de la Región de Campo Mourão), que representa a veinticinco municipios, de esta manera, la ciudad ofrece apoyo a su microrregión a través de la atención médica, la educación, la industria y otras áreas.

Figura 01. Ubicación de la Biblioteca Municipal.

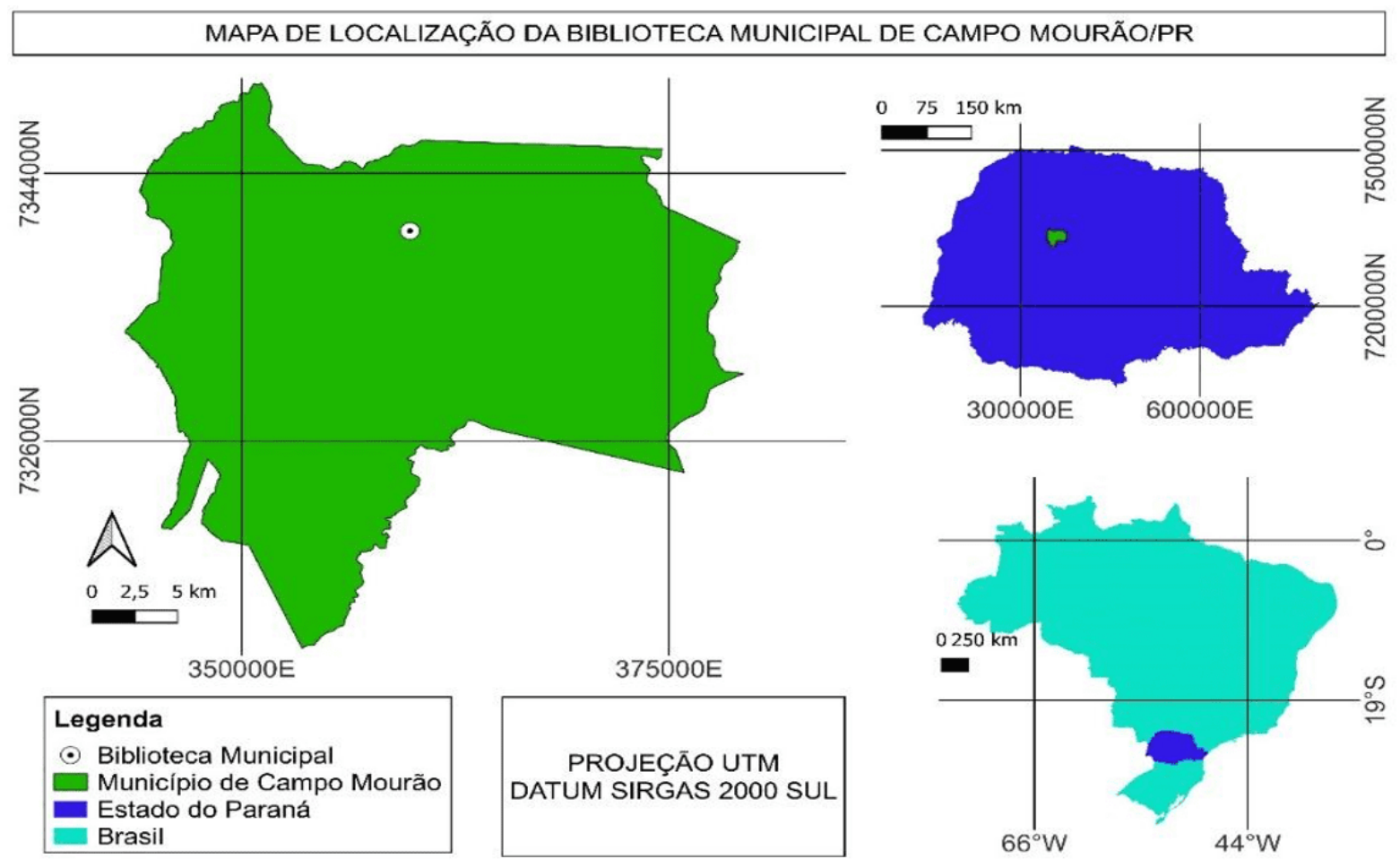

Fuente: Jairo Jerônimo de Campos Filho (2021).

El edificio en estudio tiene una gran relevancia histórica en el contexto del desarrollo del municipio, ya que originalmente fue diseñado para ser la Terminal de Autobuses.

RC: 100245

Disponible: https://www.nucleodoconhecimento.com.br/ingenieria-ambiental-es/labiblioteca-municipal 
Posteriormente, el edificio fue adaptado para albergar la biblioteca, que se inauguró el 30 de diciembre de 2004.

Figura 02. Antigua Estación de Autobuses de Campo Mourão (A), actual Biblioteca Municipal (B), Fachada Principal Biblioteca Municipal (C).

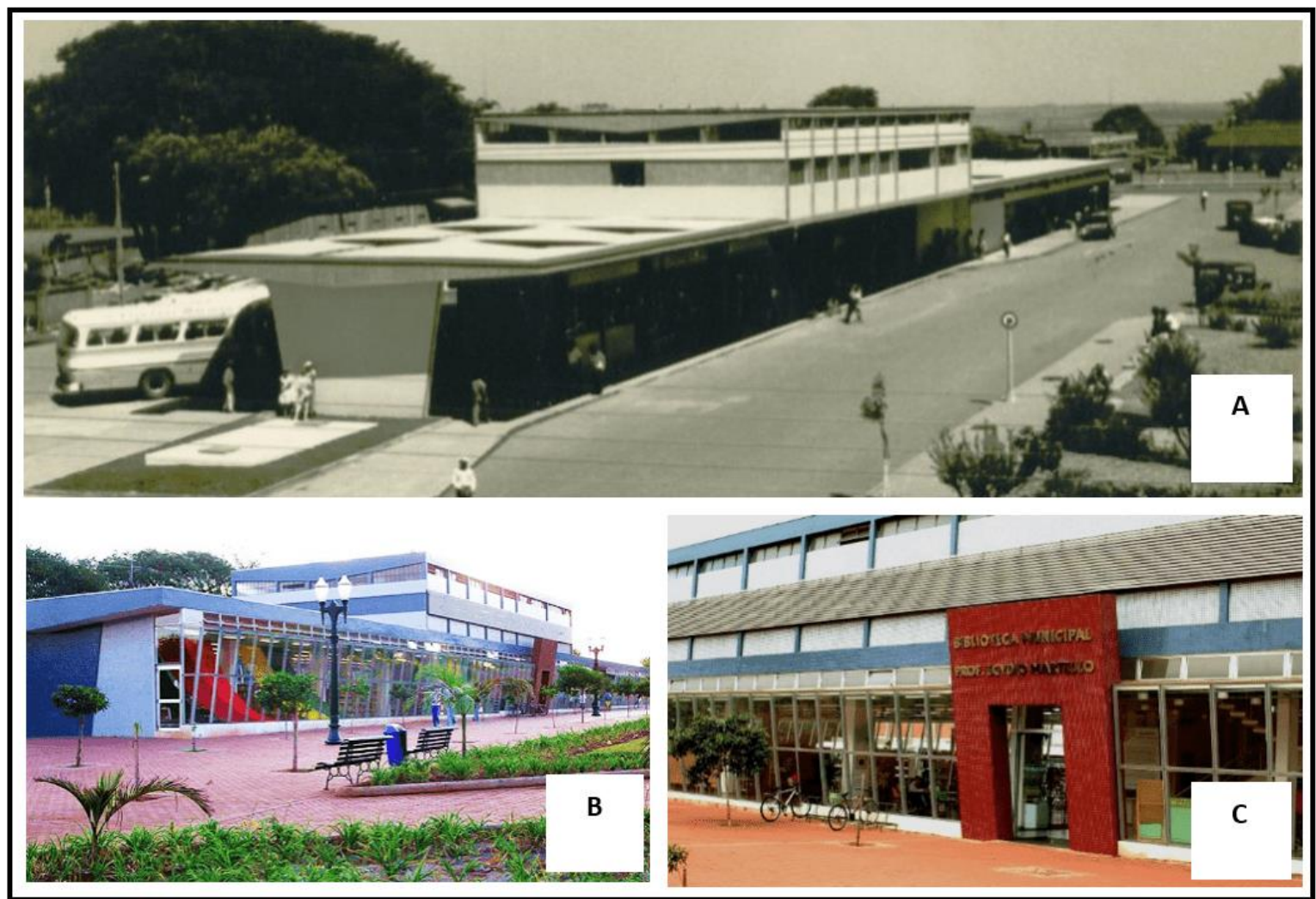

Fuente: (A) Colección personal de Jair Elias dos Santos Júnior (1969) y (B, C) colección de la Biblioteca Municipal Profesor Egydio Martello (2007).

Además de una vasta colección, espacio de exposición artística, ludoteca y áreas de estudio, la biblioteca también alberga la Academia de Letras Mourãoense. Se trata de un edificio lineal, de dos plantas, en estructura de hormigón armado, con una superficie total construida de 1772,92 m², según la disposición de la Biblioteca de la Figura 03. Gran parte de su revestimiento consiste en cortinas de vidrio translúcido, lo que favorece el calentamiento no deseado de los espacios internos. Es importante

RC: 100245

Disponible: https://www.nucleodoconhecimento.com.br/ingenieria-ambiental-es/labiblioteca-municipal 
destacar que su fachada principal está orientada hacia el noreste, por lo tanto, recibe una alta incidencia de radiación solar en el periodo estival.

Figura 03. Distribución Biblioteca Planta Baja (A), Oficinas Planta Baja (B), Planta Superior Biblioteca (C).

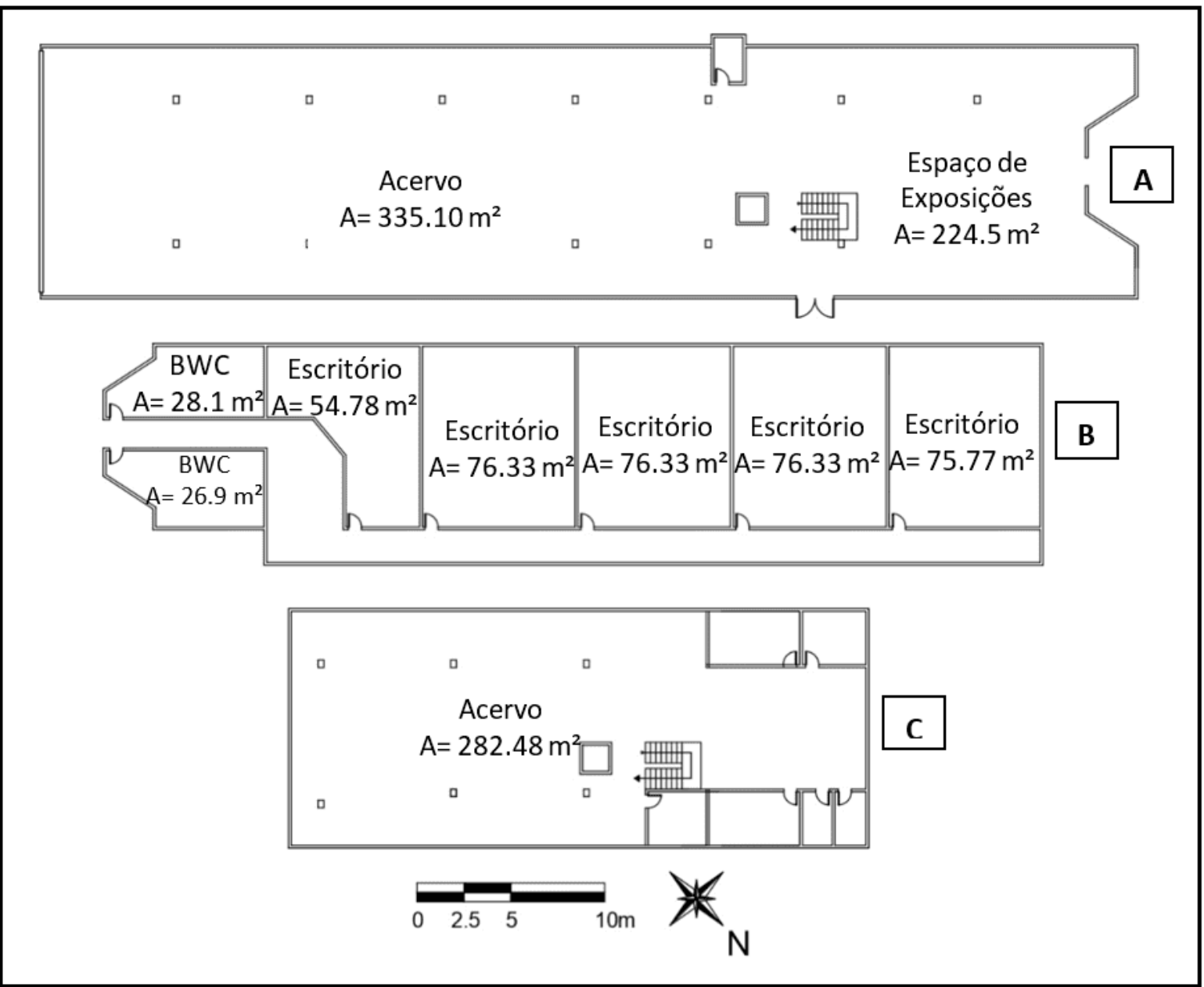

Fuente: Adaptado del Proyecto Arquitectónico original (2003).

\subsection{MÉTODOS}

El método adoptado para el presente estudio es el método prescriptivo, que se aplica solo a edificios acondicionados y evalúa la eficiencia energética a través de ecuaciones, de acuerdo con la Zona Bioclimática y el área total del edificio. Para

RC: 100245

Disponible: https://www.nucleodoconhecimento.com.br/ingenieria-ambiental-es/labiblioteca-municipal 
obtener puntuaciones para la etiqueta general de ENCE se analizaron tres sistemas: Revestimiento, Sistema de iluminación y Aire acondicionado. Esta metodología requiere la evaluación de los requisitos previos generales y específicos del sistema, que se examinarán a continuación.

\subsubsection{REQUISITOS PREVIOS GENERALES}

Para los niveles A, B y C, hay dos requisitos previos generales. El incumplimiento de los mismos afecta la puntuación final del edificio.

- El edificio debe tener un circuito eléctrico distinto para cada uso, ya sea equipo de aire acondicionado, lámparas u otros. Sin embargo, el Manual de Aplicación de RTQ-C (2017) establece que esta partida no es obligatoria para edificios construidos antes de 2009, por lo tanto, el edificio en estudio está exento de ella.

- Calentamiento de agua: Este requisito previo se aplica solo en edificios comerciales o de servicios, donde el consumo de agua caliente es superior al $10 \%$ de la demanda total de energía. En el caso del objeto de estudio, no hay instalación de calentadores de agua, por lo tanto, este elemento no se aplica.

\subsubsection{ECUACIONES PARA EL PROCEDIMIENTO DE CÁLCULO}

En el proceso de clasificación individual de los sistemas, así como para la clasificación general de la eficiencia, se utilizaron ecuaciones de acuerdo con la Tabla 01.

RC: 100245

Disponible: https://www.nucleodoconhecimento.com.br/ingenieria-ambiental-es/labiblioteca-municipal 
Tabla 01. Tabla general de ecuaciones.

\begin{tabular}{|c|c|c|}
\hline NOME & EQUAÇÃO & VARIÁVEIS \\
\hline $\begin{array}{c}\text { Equação } \\
01\end{array}$ & $\begin{array}{c}\text { IC env }=-14,14 . F A- \\
113,94 . F F+50,82 . P A F t o t a l+4,86 . F S- \\
\text { 0,32.AVS }+0,26 \cdot A H S-\frac{35,75}{F F}- \\
\text { 0,54.PAFtotal.AHS }+277,98\end{array}$ & $\begin{array}{l}\text { IC env: Indicador de Consumo da } \\
\text { Envoltória. } \\
\text { Ape: Área de projeção do edifício } \\
\left(\mathrm{m}^{2}\right) \text {; } \\
\text { Ato: Área total construída }\left(\mathrm{m}^{2}\right) ; \\
\text { Aenv: Área da envoltória }\left(\mathrm{m}^{2}\right) ; \\
\text { Apcob: Área de projeção da } \\
\text { cobertura }\left(\mathrm{m}^{2}\right) ; \\
\text { AVS: Ângulo Vertical de } \\
\text { Sombreamento } \\
\text { AHS: Ângulo Horizontal de } \\
\text { Sombreamento } \\
\text { FF: Fator de Forma, (Aenv/ Vtot); } \\
\text { FA: Fator Altura, (Apcob/ Atot); } \\
\text { FS: Fator Solar; } \\
\text { PAF } \mathrm{T} \text { : Percentual de Abertura na } \\
\text { Fachada total: média do percentual } \\
\text { de aberturas existentes; }\end{array}$ \\
\hline
\end{tabular}

RC: 100245

Disponible: https://www.nucleodoconhecimento.com.br/ingenieria-ambiental-es/labiblioteca-municipal 


\begin{tabular}{|c|c|c|}
\hline $\begin{array}{c}\text { Equação } \\
05\end{array}$ & $\begin{array}{l}\mathbf{P T}=0,3 \cdot\left\{\left(\text { EqNumEnv } \frac{A C}{A U}\right)+\right. \\
\left.\left(\frac{A P T}{A U} \cdot 5+\frac{A N C}{A U} \cdot \text { EqNu } \mathrm{mV}\right)\right\}+0,3 . \\
(\text { EqNumDPl })+0,4 \cdot\{(\text { EqNumCA } . \\
\left.\left.\frac{A C}{A U}\right)+\left(\frac{A P T}{A U} \cdot 5+\frac{A N C}{A U} \cdot \text { EqNumV }\right)\right\}+ \\
\mathrm{b}_{0}^{1}\end{array}$ & $\begin{array}{l}\text { EqNumEnv: Equivalente Numérico } \\
\text { Envoltória } \\
\text { AC: Área Condicionada } \\
\text { AU: Área útil } \\
\text { APT: Área de permanência } \\
\text { Transitória } \\
\text { ANC: Área não condicionada } \\
\text { EqNumV: Equivalente Numérico } \\
\text { Área Ventilada } \\
\text { EqNumDPI: Equivalente Numérico } \\
\text { Densidade de Potência Instalada } \\
\text { EqNumCa: Equivalente Numérico } \\
\text { Condicionamento de Ar } \\
\text { b: Bonificações }\end{array}$ \\
\hline & & $\begin{array}{l}\text { Vto }: \text { Volume total da edificação } \\
\left(\mathrm{m}^{3}\right) \text {; }\end{array}$ \\
\hline $\begin{array}{c}\text { Equação } \\
02\end{array}$ & $\mathbf{i}=\frac{I C \text { máx }-I C \text { mín }}{4}$ & $\begin{array}{l}\text { i: Intervalo } \\
\text { IC } \mathbf{C}_{\max } \text { Indicador de Consumo } \\
\text { máximo } \\
\text { IC } \mathbf{C}_{\min }: \text { Indicador de Consumo } \\
\text { Mínimo }\end{array}$ \\
\hline $\begin{array}{c}\text { Equação } \\
03\end{array}$ & $\mathbf{P}_{\text {lim }}=\mathrm{A}^{2}$. DPIL & $\begin{array}{l}\text { PI }_{\text {im: Potência Limite }} \\
\text { A: Área iluminada } \\
\text { DPIL: Densidade de Potência } \\
\text { Instalada Limite }\end{array}$ \\
\hline $\begin{array}{c}\text { Equação } \\
04\end{array}$ & EqNumCA $=\sum_{n=1}^{x}:\left[\begin{array}{l}\text { EqNumCA } \\
n\end{array} \frac{\text { Capn }}{\text { Capt }}\right]$ & $\begin{array}{l}\text { EqNumCA: Equivalente Numérico } \\
\text { de Condicionamento de } \mathrm{Ar} \\
\text { Capn: Capacidade de cada } \\
\text { sistema } \\
\text { Capt: Capacidade total dos } \\
\text { sistemas } \\
\text { x: Quantidade de sistemas }\end{array}$ \\
\hline
\end{tabular}

Fuente: Adaptado del Manual de Aplicación para RTQ-C (2017).

\subsubsection{REVESTIMIENTO}

Para cumplir con los requisitos previos específicos del sistema de Revestimiento, se deben observar los valores de las variables descritas en el Gráfico 02.

RC: 100245

Disponible: https://www.nucleodoconhecimento.com.br/ingenieria-ambiental-es/labiblioteca-municipal 
Tabla 02. Requisitos específicos para Revestimiento- Zona 3.

\begin{tabular}{|c|c|c|c|c|c|c|}
\hline Nível & \multicolumn{3}{|c|}{ Transmitância Térmica (U) } & \multicolumn{2}{|c|}{$\begin{array}{c}\text { Cores e } \\
\text { Absortância } \\
\text { Térmica }(\alpha)\end{array}$} & $\begin{array}{c}\text { Iluminação } \\
\text { Zenital }\end{array}$ \\
\hline \multirow{2}{*}{ - } & \multicolumn{2}{|c|}{ Cobertura } & Parede & Cobertura & Parede & \multirow{6}{*}{ N/A } \\
\hline & $\begin{array}{c}\text { Ambiente } \\
\text { Condicionado }\end{array}$ & $\begin{array}{l}\text { Ambiente não } \\
\text { condicionado }\end{array}$ & - & \multicolumn{2}{|l|}{-} & \\
\hline A & $1.0 \mathrm{~W} / \mathrm{m}^{2} \mathrm{~K}$ & $2.0 \mathrm{~W} / \mathrm{m}^{2} \mathrm{~K}$ & $\begin{array}{c}3.7 \\
\mathrm{~W} / \mathrm{m}^{2} \mathrm{k} \\
\end{array}$ & $<0.50$ & $<0.50$ & \\
\hline B & $1.5 \mathrm{~W} / \mathrm{m}^{2} \mathrm{k}$ & $2.0 \mathrm{~W} / \mathrm{m}^{2} \mathrm{~K}$ & $\begin{array}{c}3.7 \\
\mathrm{~W} / \mathrm{m}^{2} \mathrm{k}\end{array}$ & $<0.50$ & $\mathrm{~N} / \mathrm{A}$ & \\
\hline C & \multicolumn{2}{|c|}{$2.0 \mathrm{~W} / \mathrm{m}^{2} \mathrm{~K}$} & $\begin{array}{c}3.7 \\
\mathrm{~W} / \mathrm{m}^{2} \mathrm{k}\end{array}$ & \multirow{2}{*}{\multicolumn{2}{|c|}{$\mathrm{N} / \mathrm{A}$}} & \\
\hline E & \multicolumn{2}{|c|}{$>2.0 \mathrm{~W} / \mathrm{m}^{2} \mathrm{~K}$} & $\begin{array}{c}>3.7 \\
\mathrm{~W} / \mathrm{m}^{2} \mathrm{~K}\end{array}$ & & & \\
\hline
\end{tabular}

Fuente: Adaptado del Manual de Aplicación RTQ-C (2017).

ABNT NBR 15220-2 (2008) establece parámetros para el rendimiento térmico de los edificios. Después de verificar la asistencia de los valores anteriores de acuerdo con la norma, es necesario observar la Zona Bioclimática en la que se inserta el objeto de estudio. Según el Anexo 1 del Manual de Aplicación de RTQ-C (2017), la ciudad de Campo Mourão se encuentra en la Zona Bioclimática 3, como se muestra en la Figura 04.

RC: 100245

Disponible: https://www.nucleodoconhecimento.com.br/ingenieria-ambiental-es/labiblioteca-municipal 
Figura 04. Zonificación bioclimática de Brasil.

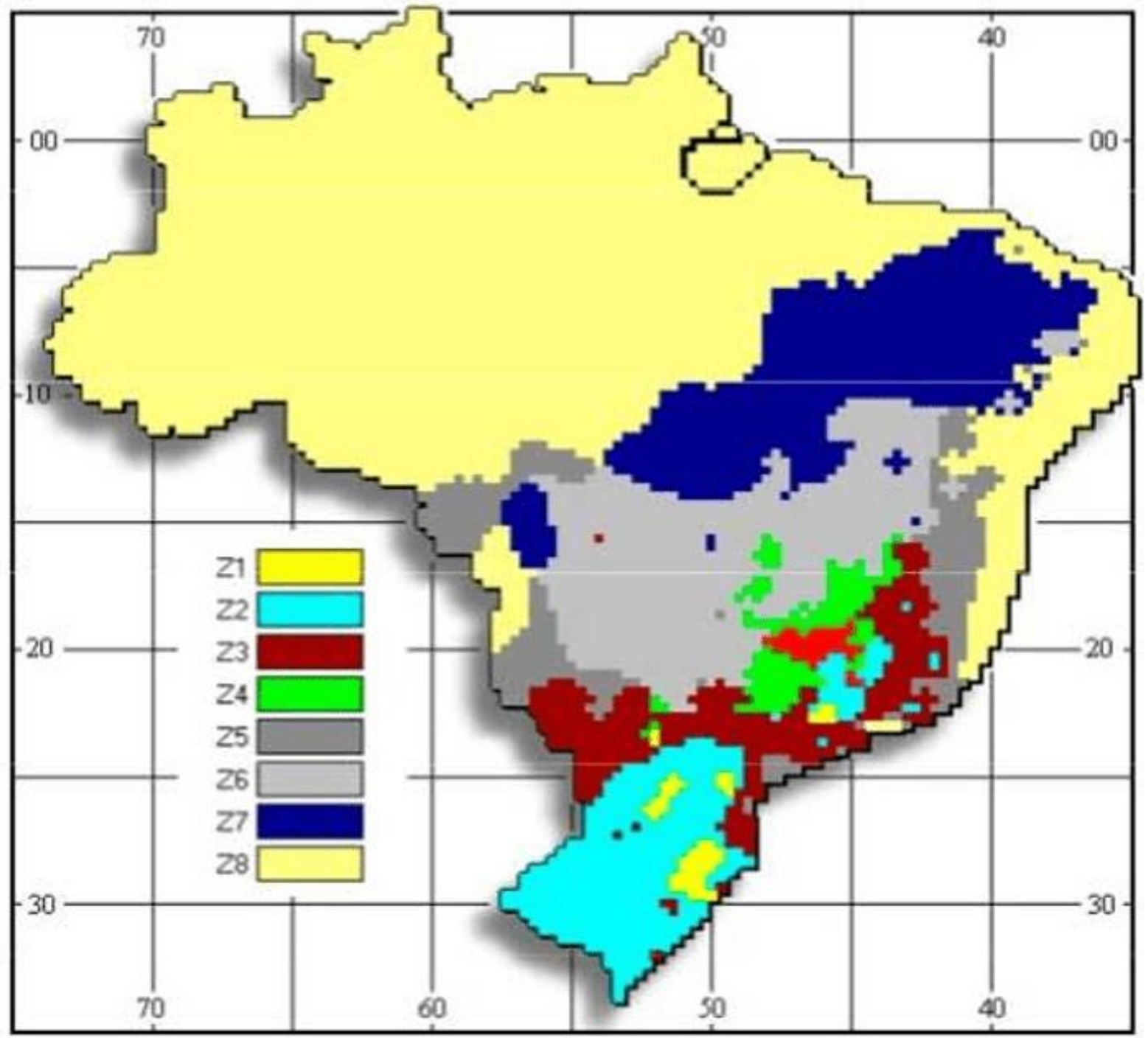

Fuente: Bioclimatismo (2021).

Para el procedimiento de cálculo fueron necesarias la información extraída del proyecto arquitectónico y las variables según la Tabla 01.

Teniendo en cuenta que el Manual especifica diferentes ecuaciones según el área del objeto de estudio, para áreas superiores a $500 \mathrm{~m}^{2}$ la ecuación 01 encaja en el edificio.

RC: 100245

Disponible: https://www.nucleodoconhecimento.com.br/ingenieria-ambiental-es/labiblioteca-municipal 
Poco después, el IC mínimo y el IC máximo se determinaron utilizando la misma ecuación, pero con los valores de entrada de acuerdo con el Gráfico 03.

Tabla 03. Parámetros de IC máximo y Cl mínimo.

\begin{tabular}{|l|c|c|c|c|}
\hline & PAF & FS & AVS & AHS \\
\hline IC máximo & 0,60 & 0,61 & 0 & 0 \\
\hline IC mínimo & 0,05 & 0,87 & 0 & 0 \\
\hline
\end{tabular}

Fuente: Manual para la aplicación de RTQ-C (2017).

Por lo tanto, el intervalo (i) de IC máximo e IC mínimo se calculó mediante la ecuación 02. Con el valor de (i), se definió la tabla 04 :

Tabla 04. Límites de los intervalos de los niveles de eficiencia.

\begin{tabular}{|c|c|c|c|c|c|}
\hline Eficiência & A & B & C & D & E \\
\hline Lim. Mín. & N/A & $\begin{array}{c}\text { IC máx }- \\
3 i+0,01\end{array}$ & $\begin{array}{c}\text { IC máx }- \\
2 i+0,01\end{array}$ & $\begin{array}{c}\text { IC máx }-\mathrm{i} \\
+0,01\end{array}$ & $\begin{array}{c}\text { IC máx }+ \\
0,01\end{array}$ \\
\hline Lim. Máx. & $\begin{array}{c}\text { IC máx }- \\
3 i\end{array}$ & IC máx $-2 \mathrm{i}$ & IC máx $-\mathrm{i}$ & IC máx & N/A \\
\hline
\end{tabular}

Fuente: Manual para la aplicación de RTQ-C (2017).

Finalmente, a partir de los valores de la Tabla 04, se comparó la tasa de revestimiento de ci con los límites de IC máximo e IC mínimo para identificar el nivel de eficiencia.

\subsubsection{SISTEMA DE ILUMINACIÓN}

El cumplimiento de los requisitos previos específicos para el sistema de iluminación se describe en el gráfico 05 .

RC: 100245

Disponible: https://www.nucleodoconhecimento.com.br/ingenieria-ambiental-es/labiblioteca-municipal 
Tabla 05. Requisitos previos para la iluminación.

\begin{tabular}{|l|l|l|l|l|}
\hline Pré-Requisito & Nível A & Nível B & Nível C & Situação \\
\hline 1. Divisão dos circuitos & Exigido & Exigido & Exigido & Isento \\
\hline 2. Contribuição da luz natural & Exigido & Exigido & N/A & $\begin{array}{l}\text { Não } \\
\text { atende }\end{array}$ \\
\hline $\begin{array}{l}\text { 3. Desligamento automático } \\
\text { do sistema de iluminação }\end{array}$ & Exigido & N/A & N/A & $\begin{array}{l}\text { Não } \\
\text { atende }\end{array}$ \\
\hline
\end{tabular}

Fuente: Adaptado del Manual de Aplicación RTQ-C (2017).

El edificio en estudio está exento del requisito previo número 1, y no cumple con los otros requisitos previos, por lo que el nivel del sistema de iluminación solo puede tener automáticamente como clasificación máxima el nivel C. Sin embargo, su clasificación final está sujeta a los cálculos del Límite de Densidad de Potencia Instalada.

Hay dos métodos para calcular la eficiencia del sistema de iluminación:

- Método de Área: Evalúa conjuntamente ambientes (para edificios con 3 actividades principales, o actividades que ocupan más del 30\% del área construida).

- Método de actividad: Evalúa individualmente cada actividad.

Dado que la Biblioteca Municipal alberga 3 actividades principales, y ocupan más del $30 \%$ del área total, optamos por el Método área. El procedimiento de cálculo se llevó a cabo a partir de los siguientes pasos:

a) Las principales actividades se identificaron de acuerdo con la Tabla de Límite Máximo Aceptable de Densidad de Potencia de lluminación (DPIL) para el nivel de eficiencia deseado - Método del área de construcción, del Manual de Aplicación de RTQ-C (2017). Para las actividades no descritas, se eligió una actividad equivalente.

RC: 100245

Disponible: https://www.nucleodoconhecimento.com.br/ingenieria-ambiental-es/labiblioteca-municipal 
b) Se identificó el área de cada actividad, en metros cuadrados.

c) A continuación, se definió la potencia límite para cada actividad, multiplicando el área iluminada por el DPIL proporcionado en la misma Tabla del Manual, según la Ecuación 03. A partir de la potencia límite para cada actividad, la suma de las potencias determinó la potencia límite del edificio.

d) Finalmente, se comparó la potencia total instalada en el edificio con la potencia límite para determinar el nivel de eficiencia del sistema de iluminación utilizando el Equivalente Numérico proporcionado en la Tabla 06.

Tabla 06. Equivalente numérico (EqNum) para cada nivel de eficiencia.

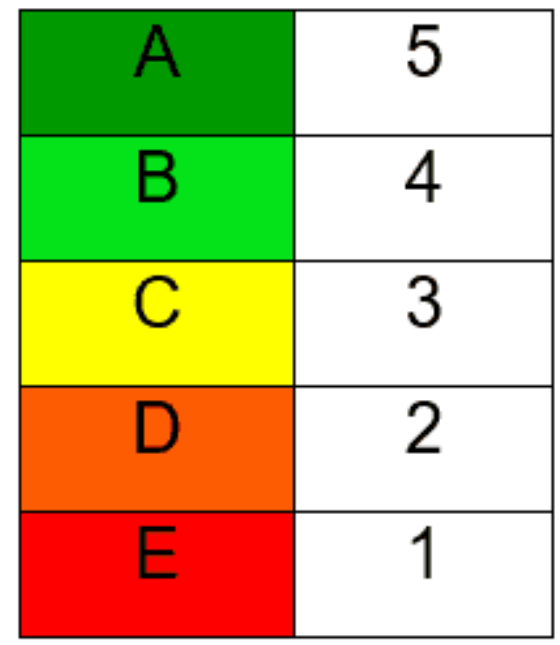

Fuente: Manual de aplicación de RTQ-C (2017).

\subsubsection{AIRE ACONDICIONADO}

Los equipos de climatización instalados en la Biblioteca Municipal están certificados por el INMETRO, por lo que se utilizó la información técnica proporcionada por el propio instituto y los fabricantes. El procedimiento para determinar la eficiencia del sistema se segregó en etapas:

RC: 100245

Disponible: https://www.nucleodoconhecimento.com.br/ingenieria-ambiental-es/labiblioteca-municipal 
a) La eficiencia de cada sistema se observó a través de la etiqueta en el equipo.

b) El coeficiente de ponderación se determinó mediante la relación entre la potencia de cada unidad y la suma de las potencias de todas las unidades.

c) Del Coeficiente de Ponderación multiplicado por el Equivalente Numérico según la Tabla 06, se obtuvo el Resultado Ponderado.

d) La suma de los Resultados Ponderados de cada unidad determinó la eficiencia total, expresada en la Ecuación 04.

Debido a que existen distintas eficiencias, el sistema de Aire Acondicionado debe tener su Equivalente Numérico de acuerdo a la Tabla 07:

Tabla 07. Equivalente numérico para sistemas de aire acondicionado y puntuación general.

\begin{tabular}{|c|c|}
\hline$A$ & $>4,5$ a 5 \\
\hline$B$ & $>3,5 a<4,5$ \\
\hline$C$ & $>2,5 a<3,5$ \\
\hline$D$ & $>1,5 a<2,5$ \\
\hline$E$ & $<1,5$ \\
\hline
\end{tabular}

Fuente: Manual de aplicación de Rtq-C (2017).

RC: 100245

Disponible: https://www.nucleodoconhecimento.com.br/ingenieria-ambiental-es/labiblioteca-municipal 


\subsubsection{PUNTUACIÓN TOTAL}

La determinación del nivel de eficiencia total del edificio se expresa a través de la Ecuación 05, que asigna pesos para cada sistema: $30 \%$ para revestimiento, $30 \%$ para sistema de iluminación y $40 \%$ para sistema de aire acondicionado. A partir del resultado de la ecuación, es necesario convertir el valor numérico determinado para cada nivel, como se muestra en la Tabla 07.

\section{RESULTADOS Y DISCUSIÓN}

\subsection{REVESTIMIENTO}

Para analizar la revestimiento, primero se observó el cumplimiento de los requisitos previos específicos para este sistema. La Tabla 08 muestra la puntuación para cada requisito previo.

Tabla 08. Cumplimiento de requisitos previos específicos.

\begin{tabular}{|c|c|c|c|c|}
\hline \multirow{4}{*}{ Nível } & \multicolumn{2}{|c|}{ Transmitância Térmica (U) } & \multicolumn{2}{|c|}{$\begin{array}{c}\text { Cores e Absortância } \\
\text { Térmica }(\alpha)\end{array}$} \\
\hline & Cobertura & Parede & Cobertura & Parede \\
\hline & $\begin{array}{c}\text { Telha } \\
\text { fibrociment } \\
\text { o } 0.7 \mathrm{~cm} \mathrm{U} \\
=1.75 \mathrm{Wm}^{-} \\
{ }^{2} \mathrm{~K}\end{array}$ & $\begin{array}{c}\text { Tijolo } 6 \text { furos } \\
\text { circulares, } \\
\text { assentados na } \\
\text { maior dimensão. } U \\
=1.88 \mathrm{Wm}^{-2} \mathrm{~K}\end{array}$ & $\begin{array}{l}\text { Telha } \\
\text { fibrociment } \\
\text { o } 0.7 \mathrm{~cm} \alpha \\
=0.30\end{array}$ & $\begin{array}{c}\text { Vidro Incolor } \\
\alpha=0.25\end{array}$ \\
\hline & C & A & A & $A$ \\
\hline
\end{tabular}

Fuente: Autoría propia.

A continuación, se determinó el Indicador de Consumo (IC) según la Ecuación 01. La Tabla 09 indica los valores para cada variable, obtenidos del proyecto arquitectónico.

RC: 100245

Disponible: https://www.nucleodoconhecimento.com.br/ingenieria-ambiental-es/labiblioteca-municipal 
Tabla 09. Variables del proyecto.

\begin{tabular}{|l|c|}
\hline \multicolumn{1}{|c|}{ Variáveis de Projeto } & Valores \\
\hline Ape: Área de projeção do edifício $\left(\mathrm{m}^{2}\right) ;$ & $799.97 \mathrm{~m}^{2}$ \\
\hline Atot: Área total construída $\left(\mathrm{m}^{2}\right) ;$ & $1599.95 \mathrm{~m}^{2}$ \\
\hline Aenv: Área da envoltória $\left(\mathrm{m}^{2}\right) ;$ & $2641.75 \mathrm{~m}^{2}$ \\
\hline Apcob: Área de projeção da cobertura $\left(\mathrm{m}^{2}\right) ;$ & $1280.13 \mathrm{~m}^{2}$ \\
\hline AVS: Ângulo Vertical de Sombreamento & $0^{*}$ \\
\hline AHS: Ângulo Horizontal de Sombreamento; & $0^{*}$ \\
\hline FF: Fator de Forma, (Aenv/ Vtot); & 0.401 \\
\hline FA: Fator Altura, (Apcob/ Atot); & 0.194 \\
\hline FS: Fator Solar; & 0.27 \\
\hline $\begin{array}{l}\text { PAFT: Percentual de Abertura na Fachada total: média do } \\
\text { percentual de aberturas existentes; }\end{array}$ & $34.76 \%$ \\
\hline Vtot: Volume total da edificação $(\mathrm{m} 3) ;$ & $6575.15 \mathrm{~m}^{3}$ \\
\hline
\end{tabular}

Fuente: Autoría propia.

* Valores nulos, porque el edificio no presenta aleros, ni elementos verticales de protección solar.

Para determinar el Factor Solar, se utilizó el vidrio comercial estándar para edificios Cool Lite 114 PN 8 mm, según lo especificado en el Catálogo de Propiedades Térmicas y Ópticas de Vidrios Comercializados en Brasil. Después del cálculo, se obtuvo como resultado el Indicador de Consumo Revestimiento (IC) igual a 159.373.

Luego, se calcularon el IC máximo (IC $\mathrm{Cmax}_{\max }$ y el IC mínimo ( $\left(\mathrm{C}_{\min }\right)$, también utilizando la Ecuación 01, pero sustituyendo los valores descritos en la Tabla 04. Como resultado, se concluyó que IC $\max$ es igual a 173.852 y IC $\min$ es igual a 147.165.

Para calcular el Intervalo (i) IC $\mathrm{C}_{\max }$ e IC $\min$ se aplicó la Ecuación 02, donde el resultado obtenido fue 6.671 .

RC: 100245

Disponible: https://www.nucleodoconhecimento.com.br/ingenieria-ambiental-es/labiblioteca-municipal 
A partir del resultado de (i), los valores de (i) de la Tabla 05 fueron reemplazados por 6.671, dando como resultado la Tabla 10, que presenta los límites mínimos y máximos para cada nivel de eficiencia.

Tabla 10. Valores ICenv máximo y mínimo para cada nivel.

\begin{tabular}{|c|c|c|c|c|c|}
\hline \multirow{5}{*}{ Mínimo } & A & B & C & D & E \\
\hline & \multirow{4}{*}{ N/A } & $\begin{array}{c}\text { IC máx }-3 i+ \\
0,01\end{array}$ & $\begin{array}{c}\text { IC máx }-2 i+ \\
0,01\end{array}$ & $\begin{array}{c}\text { IC máx - i + } \\
0,01\end{array}$ & IC máx $+0,01$ \\
\hline & & $\begin{array}{c}173,852-3 \\
(6,671)+ \\
0,01\end{array}$ & $\begin{array}{c}173,852-2 \\
(6,671)+ \\
0,01\end{array}$ & $\begin{array}{c}173,852- \\
6,671+0,01\end{array}$ & $173,852+0,01$ \\
\hline & & $\begin{array}{c}173,852- \\
20,013+ \\
0,01\end{array}$ & $\begin{array}{c}173,852- \\
13,342+ \\
0,01\end{array}$ & \multirow[b]{2}{*}{167,191} & \multirow[b]{2}{*}{173,862} \\
\hline & & *153,849 & 160,52 & & \\
\hline \multirow{5}{*}{ Máximo } & A & $\mathbf{B}$ & C & D & E \\
\hline & IC máx - 3i & IC máx - 2i & IC máx - i & IC máx & \multirow{4}{*}{$\mathrm{N} / \mathrm{A}$} \\
\hline & $\begin{array}{c}173,852-3 \\
(6,671)\end{array}$ & $\begin{array}{c}173,852-2 \\
(6,671)\end{array}$ & $\begin{array}{c}173,852- \\
6,671 \\
\end{array}$ & \multirow[b]{3}{*}{173.852} & \\
\hline & $\begin{array}{c}173,852- \\
20,013\end{array}$ & $\begin{array}{c}173,852- \\
13,342 \\
\end{array}$ & \multirow[b]{2}{*}{167,181} & & \\
\hline & 153,839 & *160,51 & & & \\
\hline
\end{tabular}

Fuente: Autoría propia.

* Valor real de la biblioteca IC env con los límites encontrados, donde el nivel de eficiencia de la envolvente es B $(160,51<159,373>153,849)$.

\subsection{SISTEMA DE ILUMINACIÓN}

Para determinar el nivel de eficiencia del Sistema de lluminación utilizando el Método de Área, se identificaron las actividades desarrolladas en cada espacio de la Biblioteca. El cumplimiento de los requisitos previos se observó individualmente para cada entorno, finalmente, el análisis del sistema en su conjunto se realizó a través de la suma del DPIL de todos los entornos. El procedimiento de cálculo se basó en la información contenida en el cuadro 11.

RC: 100245

Disponible: https://www.nucleodoconhecimento.com.br/ingenieria-ambiental-es/labiblioteca-municipal 
Tabla 11. Clasificación del sistema de iluminación.

\begin{tabular}{|c|c|c|c|c|c|}
\hline \multicolumn{2}{|l|}{ Atividade } & $\begin{array}{c}\text { Biblioteca } 1^{\circ} \\
\text { Pavimento }\end{array}$ & $\begin{array}{c}\text { Biblioteca } 2^{\circ} \\
\text { Pavimento }\end{array}$ & Escritório & $\begin{array}{c}\text { Total } \\
\text { Edifício* }\end{array}$ \\
\hline \multicolumn{2}{|l|}{ Área lluminada $\left(\mathrm{m}^{2}\right)$} & 559.6 & 282.48 & 359.63 & 1201.71 \\
\hline \multirow{4}{*}{ DPIL RTQ-C } & A & \multicolumn{2}{|c|}{12.7} & 9.7 & \multirow{4}{*}{$N / A$} \\
\hline & B & \multicolumn{2}{|c|}{14.6} & 11.2 & \\
\hline & C & \multirow{2}{*}{\multicolumn{2}{|c|}{16.5}} & 12.6 & \\
\hline & D & & & 14.1 & \\
\hline \multirow{2}{*}{ Lâmpadas } & $\begin{array}{l}40 \\
W\end{array}$ & 90 units & 104 units & 146 units & 340 \\
\hline & $\begin{array}{l}18 \\
W\end{array}$ & 142 units & 0 & 0 & 142 \\
\hline \multicolumn{2}{|c|}{ Potência Instalada (W) } & 6156 & 4160 & 5840 & 16156 \\
\hline \multirow{4}{*}{ Potência Limite (W) } & A & 7106.92 & 3587.49 & 3488.41 & 14182.82 \\
\hline & B & 8170.16 & 4124.2 & 4027.85 & 16322.21 \\
\hline & C & 9233.4 & 4660.92 & 4531.33 & 18425.65 \\
\hline & D & 10296.64 & 5197.63 & 5070.78 & 20565.05 \\
\hline \multicolumn{2}{|l|}{ Classificação Edifício } & \multicolumn{4}{|c|}{ Nível B } \\
\hline
\end{tabular}

Fuente: Autoría propia.

* El Área Total lluminada del Edificio en metros cuadrados representa solo las áreas de estadía prolongada, es decir, no incluye áreas secundarias como, baños, dosel, circulación y otras. Dado que las ecuaciones descritas en el Manual para la Aplicación de RTQ-C ya predicen un margen considerando estas áreas secundarias.

De acuerdo con los cálculos DPIL de cada ambiente según la Ecuación 03, el Sistema de lluminación se clasifica como nivel B. Sin embargo, teniendo en cuenta que el edificio en estudio no cumple con los requisitos previos según el Gráfico 05, la puntuación final del Sistema de lluminación desciende al Nivel C.

\subsection{AIRE ACONDICIONADO}

Para determinar la eficiencia del sistema de Aire Acondicionado, se realizaron los cálculos de acuerdo a la metodología, y considerando que todos los equipos de aire

RC: 100245

Disponible: https://www.nucleodoconhecimento.com.br/ingenieria-ambiental-es/labiblioteca-municipal 
acondicionado de la Biblioteca son de tipo techo partido, se obtuvieron los siguientes resultados como se muestra en el Gráfico 12.

Tabla 12. Clasificación del sistema de aire acondicionado.

\begin{tabular}{|c|c|c|c|c|c|c|}
\hline Ambiente & $\begin{array}{c}\text { Biblioteca 10 } \\
\text { Pavimento }\end{array}$ & $\begin{array}{c}\text { Biblioteca 2 } \\
\text { Pavimento }\end{array}$ & \multicolumn{3}{|c|}{ Escritório } \\
\hline Fabricante & ELGIN & ELGIN & GREE & RHEEM & KOMECO & AUSTIN \\
\hline Quantidade & 3 & 2 & 2 & 2 & 1 & 1 \\
\hline $\begin{array}{c}\text { Potência } \\
\text { (Btu/h) }\end{array}$ & 60000 & 60000 & 9000 & 20000 & 57000 & 60000 \\
\hline $\begin{array}{c}\text { Classificação } \\
\text { Energética }\end{array}$ & $\mathrm{C}$ & $\mathrm{C}$ & $\mathrm{A}$ & $\mathrm{D}$ & $\mathrm{D}$ & $\mathrm{D}$ \\
\hline $\begin{array}{c}\text { Equivalente } \\
\text { Numérico }\end{array}$ & 3 & 3 & 5 & 2 & 2 & 2 \\
\hline $\begin{array}{c}\text { Coeficiente } \\
\text { de }\end{array}$ & 0.22 & 0.22 & 0.03 & 0.07 & 0.21 & 0.22 \\
\hline $\begin{array}{c}\text { Ponderação } \\
\text { Resultado } \\
\text { Ponderado }\end{array}$ & 0.66 & 0.66 & 0.15 & 0.14 & 0.42 & 0.66 \\
\hline
\end{tabular}

Fuente: Autoría propia.A partir de la aplicación de la Ecuación 04, el resultado obtenido fue de 2,69, lo que, según la Tabla 07, equivale al nivel $C$ de eficiencia.

\subsection{PUNTUACIÓN TOTAL (PT)}

Para definir el nivel general de eficiencia energética del edificio, los resultados de cada sistema individual se sintetizaron en la Ecuación 05. La Tabla 13 muestra los valores para cada variable utilizada.

RC: 100245

Disponible: https://www.nucleodoconhecimento.com.br/ingenieria-ambiental-es/labiblioteca-municipal 
Tabla 13. Variables de ecuación 05.

\begin{tabular}{|l|c|}
\hline EqNumEnv & 4 \\
\hline AC & $1201,71 \mathrm{~m}^{2}$ \\
\hline AU & $1592,73 \mathrm{~m}^{2}$ \\
\hline APT & $182,86 \mathrm{~m}^{2}$ \\
\hline ANC & $\mathrm{N} / \mathrm{A}^{*}$ \\
\hline EqNumV & $\mathrm{N} / \mathrm{A}^{*}$ \\
\hline EqNumDPI & 4 \\
\hline EqNumCA & 3 \\
\hline B & ${\mathrm{N} / \mathrm{A}^{*}}$ \\
\hline
\end{tabular}

Fuente: Autoría propia.

* Las variables no se aplican a entornos condicionados.

El resultado PT fue de 3.111, dentro del nivel $\mathrm{C}$ de eficiencia energética.

\section{CONSIDERACIONES FINALES}

Se concluye que la Biblioteca Municipal de Campo Mourão no presenta resultados satisfactorios de eficiencia energética. Para optimizar su eficiencia, se sugiere:

- Intervención en el revestimiento con instalación de elementos de protección solar, como aleros horizontales y verticales, brises, cobogós, entre otros.

- En relación con el Sistema de lluminación, se recomienda dividir los circuitos, de manera que permita el apagado de lámparas cerca de las ventanas para el uso de luz natural.

- En cuanto al Sistema de Aire Acondicionado, es importante reemplazar el equipo actual que no tiene eficiencia $A$, ya que el $36 \%$ de los equipos tiene una calificación D.

RC: 100245

Disponible: https://www.nucleodoconhecimento.com.br/ingenieria-ambiental-es/labiblioteca-municipal 
- Además de estas medidas, también se recomienda la sustitución de baldosas de fibrocemento por baldosas termoacústicas.

- Para hacer el edificio más sostenible, es recomendable instalar placas fotovoltaicas para la generación de energía solar, ya que la inversión inicial se compensa a corto plazo, aportando ahorro, bajo mantenimiento y funcionalidad.

\section{REFERENCIAS}

ASSOCIAÇÃO BRASILEIRA DE NORMAS TÉCNICAS. NBR 15220-2: Desempenho térmico de edificações: Métodos de cálculo da transmitância térmica, da capacidade térmica, do atraso térmico e do fator solar de elementos e componentes de edificações. 2008.

CONSELHO DE ARQUIETURA E URBANISMO DO BRASIL (Brasil). Governo torna obrigatório que edifícios públicos federais tenham energia eficientes. Disponível em: https://www.caubr.gov.br/governo-torna-obrigatorio-que-edificiospublicos-federais-sejam-energeticamente-eficientes/\#comments. Acesso em: 28 ago. 2021.

CONFERÊNCIA DE ESTUDOS EM ENERGIA ELÉTRICA. PROGRAMA BRASILEIRO DE ETIQUETAGEM: Breve Panorama. Uberlândia, MG: Universidade Federal de Uberlândia, 2019. 5 p. Disponível em: https://www.lunasat.top/ceel/evento/artigos/\#2019. Acesso em: 28 ago. 2021.

CENTRO BRASILEIRO DE EFICIÊNCIA ENERGÉTICA EM EDIFICAÇÕES. Catálogo de Propriedades Térmicas e Óticas de Vidros Comercializados No Brasil. Brasil: Universidade Federal de Santa Catarina, 2015. Disponível em: https://cb3e.ufsc.br/sites/default/files/projetos/etiquetagem/catalogopropriedades-vidros-comercializados-brasil-13032015_v2.pdf. Acesso em: 28 jul. 2021.

RC: 100245

Disponible: https://www.nucleodoconhecimento.com.br/ingenieria-ambiental-es/labiblioteca-municipal 
DOS SANTOS JUNIOR, J.E. Antiga Rodoviária de Campo Mourão-PR. 1969. Fotografia.

FILHO, Jairo Jerônimo De Campos. Mapa Localização Biblioteca. Campo Mourão: 2021. Mapa em escala gráfica. Software QGIS V. 3.2.

Programa Nacional De Conservação De Energia Elétrica. Manual para aplicação do RTQ-C. 4 ed. Brasil, 2017.

HARADA, Magali. Bioclimatismo: Blog de Arquitetura Bioclimática. Disponível em: http://bioclimatismo.com.br/bioclimatismo/zoneamento-bioclimatico-brasileiro/. Acesso em: 05 jun. 2021.

IBGE - INSTITUTO BRASILEIRO DE GEOGRAFIA E ESTATÍSTICA. Censo Brasileiro de 2020. Rio de Janeiro. 2021.

DEBATES INTERDISCIPLINARES VII. O Papel das Conferências Internacionais Sobre o Meio Ambiente Para o Desenvolvimento dos Regimes Internacionais Ambientais: De Estocolmo A Rio +20. Palhoça, Sc: Unisul, 2016. Disponível em: https://www.researchgate.net/profile/Jose-Baltazar-Andrade-

Guerra/publication/301626018_Debates_Interdisciplinares_VII/links/571e496f08aeac ed7889df5e/Debates-Interdisciplinares-VII.pdf\#page=168. Acesso em: 20 ago. 2021.

Enviado: Agosto de 2021.

Aprobado: Octubre de 2021.

RC: 100245

Disponible: https://www.nucleodoconhecimento.com.br/ingenieria-ambiental-es/labiblioteca-municipal 